\title{
Immune checkpoint inhibitors in sarcomas: in quest of predictive biomarkers
}

\author{
Robin Veenstra, Marie Kostine, Anne-Marie Cleton-Jansen, Noel FCC de Miranda and Judith VMG Bovée
}

Sarcomas are a rare group of tumors of mesenchymal origin. Metastatic sarcomas are often difficult to treat and unresponsive to standard radio- and chemotherapy, resulting in a poor survival rate for patients. Novel treatments with immune checkpoint inhibitors have been proven to prolong survival of patients with a variety of cancers, including metastatic melanoma, lung, and renal cell carcinoma. Since immune checkpoint inhibitors could provide a novel treatment option for patients with sarcomas, clinical trials investigating their efficacy in these group of tumors are ongoing. However, the discrimination of patients that are the most likely to respond to these treatments is still an obstacle in the design of clinical trials. In this review, we provide a brief overview of the mechanisms of action of immune checkpoint inhibitors and discuss the proposed biomarkers of therapy response, such as lymphocytic infiltration, intratumoral PD-L1 expression, and mutational load in sarcomas.

Laboratory Investigation (2018) 98, 41-50; doi:10.1038/labinvest.2017.128; published online 20 November 2017

Sarcomas are a heterogeneous group of rare neoplasms, originating from somatic mesenchymal tissues, with more than 50 distinct histologic subtypes. Although accounting for $<1 \%$ of all adult solid malignant cancers, sarcomas form more than $20 \%$ of all pediatric solid malignant cancers. ${ }^{1}$ In spite of chemotherapy and radiotherapy, the median survival for metastatic sarcoma is $\sim 12$ months. ${ }^{2}$ Novel treatment options for these patients are therefore of utmost importance.

One class of such potential new therapeutics is the immune checkpoint inhibitors. In 2010, the treatment of advanced melanoma patients with antibodies that block cytotoxic T-lymphocyte-associated antigen 4 (CTLA-4) marked the beginning of the successful employment of this new class of immunotherapeutics. ${ }^{3}$ Added to dacarbazine, ipilimumab has doubled the 5-year survival rates in patients with advanced melanoma compared with patients treated with dacarbazine alone. ${ }^{4}$ A few years later, antibodies directed against programmed death 1 (PD-1) or PD-ligand 1 (PD-L1) demonstrated clinical efficacy in melanoma patients and in other cancer types. ${ }^{5-10}$ Moreover, therapies targeting the PD-1/PD-L1 axis produced less immune-related side effects compared to treatment with anti-CTLA-4 antibodies. ${ }^{11}$

These promising immunotherapies may offer new treatment options for sarcoma patients and clinical trials are being performed to explore their potential. Because of the heterogeneity of sarcomas, researchers and clinicians tried to identify which subtypes would be suitable for immunotherapeutic strategies. Considering the genetic background of sarcomas, it was postulated that high-grade sarcomas with complex genomes would be the best candidates for treatment with immune checkpoint inhibitors. ${ }^{12}$

Recently, biomarkers predictive of response to immune checkpoint inhibitors have been proposed and investigated and could therefore guide selection of sarcoma patients for clinical trials ${ }^{13,14}$. In this review, we describe the mechanisms of action of immune checkpoint inhibitors and discuss and summarize the literature on the presence of biomarkers in sarcomas that may predict treatment response. In light of these, we discuss the potential application of immune checkpoint inhibitors in the treatment of sarcomas.

\section{MODE OF ACTION OF IMMUNE CHECKPOINT INHIBITORS Anti-CTLA-4 Antibodies}

Our knowledge on how the immune system combats cancer cells has increased ever since Burnet ${ }^{15}$ and Thomas proposed the concept of cancer immune surveillance in the 1950s. To induce an immune response, professional antigen-presenting cells (APCs), most importantly dendritic cells (DCs), take up tumor-associated antigens and migrate via lymphatic vessels to the regional lymph nodes. ${ }^{16}$ In the lymph nodes, the DCs can activate naive $\mathrm{T}$ cells by presenting tumor antigens in complex with human leukocyte antigen (HLA) class I and II 
a

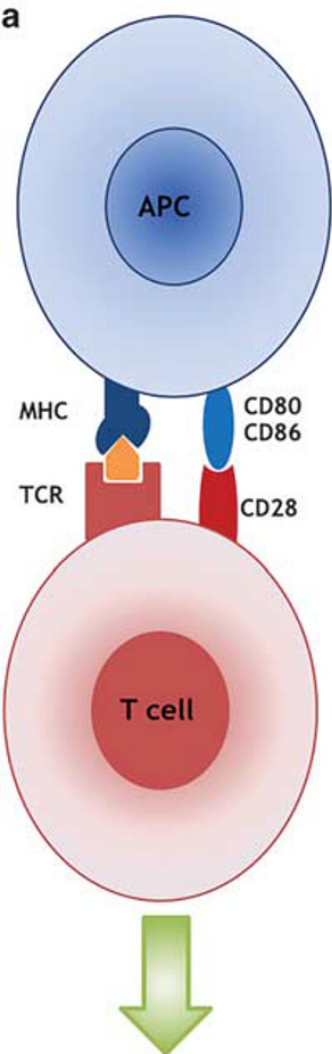

T cell activation b

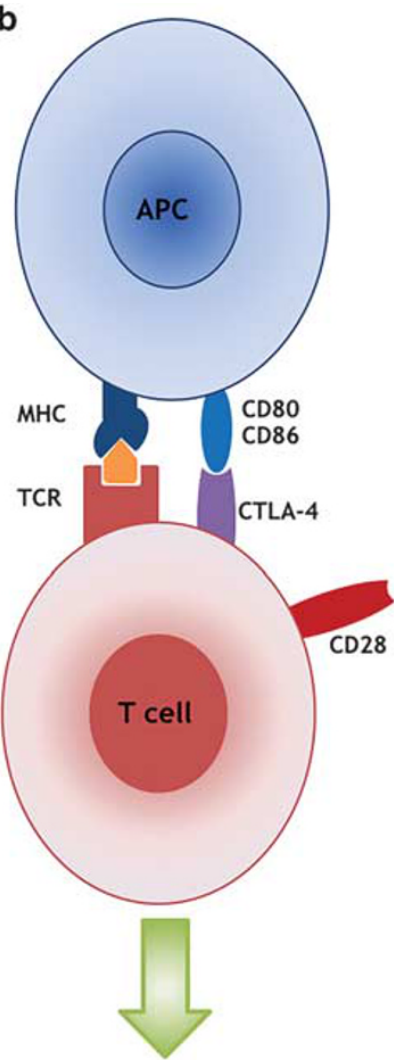

No $T$ cell activation

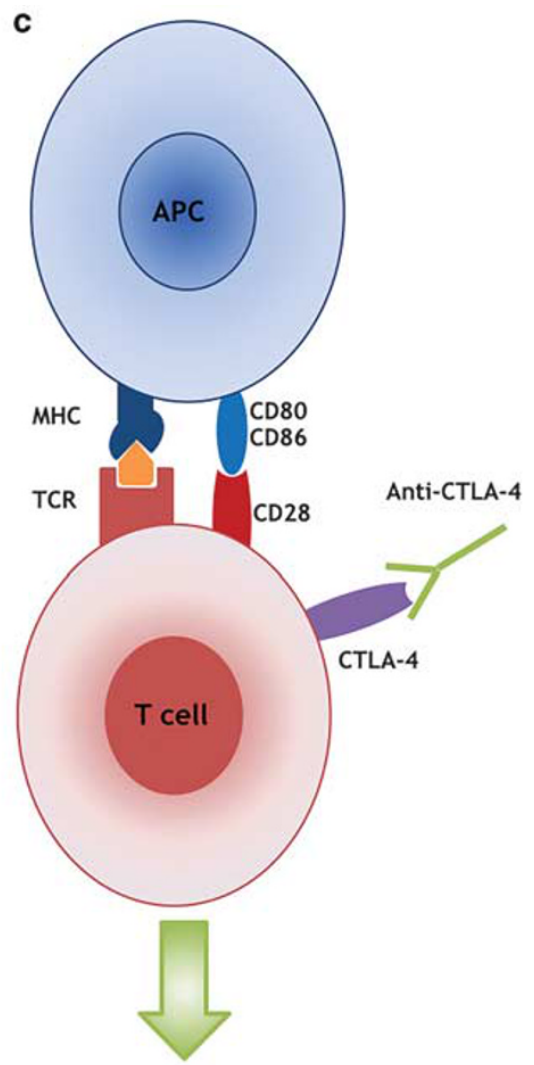

T cell activation

Figure 1 Schematic overview of the mechanism of action of anti-CTLA-4 antibodies. APCs take up tumor antigens in the periphery and migrate to the lymph nodes. In the lymph nodes, the APCs present tumor antigens in complex with HLA class I molecules to naive T cells, which then become primed. Complete activation of the naive T cell occurs when a co-stimulatory signal is provided by binding of CD80 or CD86 on the APC to CD28 on the naive T cell (a). However, naive T cells can also express CTLA-4 that, by interacting with CD80 and CD86, transduces an inhibitory signal that leads to T-cell anergy (b). Treatment with CTLA-4-blocking antibodies promotes activation of naive T cells, which can then migrate to tumor tissues and directly kill tumor cells $\left(C D 8^{+} T\right.$ cells) or provide an inflammatory environment $\left(T_{H} 1 C^{2} 4^{+}\right.$cells) (c).

molecules to the $\mathrm{T}$-cell receptor of naive $\mathrm{CD} 8^{+}$and $\mathrm{CD} 4^{+}$ $\mathrm{T}$ cells, respectively. This priming of the $\mathrm{T}$ cells requires additional co-stimulatory signals by binding of $\mathrm{CD} 28$ on the naive $\mathrm{T}$ cell to CD80 and CD86 on the APC (Figure 1a). ${ }^{17}$ The immune checkpoint molecule CTLA-4, however, can compete with CD28 for binding to CD80 and CD86, and produce inhibitory signals to the activated T cells, acting as a real break and leading to T-cell anergy and apoptosis. ${ }^{18}$ Interestingly, regulatory $\mathrm{T}$ cells ( $\mathrm{T}_{\text {reg }}$ cells) also need CTLA-4 for their immunosuppressive function, suggesting that anti-CTLA-4 treatment might also interfere with the immunosuppressive function of $\mathrm{T}_{\text {reg }}$ cells. ${ }^{19}$ The importance of CTLA-4 in maintaining a balanced immune response has been demonstrated with CTLA-4 knockout mice that develop severe autoimmune lymphoproliferative disease. ${ }^{20,21}$ By releasing such an immunosuppressive brake, treatment with antiCTLA-4 antibodies result in an enhanced activation of naive $\mathrm{T}$ cells. Although APCs can activate both $\mathrm{CD}^{+}$and $\mathrm{CD} 8^{+}$ $\mathrm{T}$ cells, treatment with anti-CTLA-4 antibodies is believed to mainly regulate the activity of $\mathrm{CD}^{+} \mathrm{T}$ cells, which can develop in Th1 $\mathrm{CD}^{+}$cells after activation and provide important cytokines, such as IL-2, for the activation of $\mathrm{CD}^{+}$ $\mathrm{T}$ cells. These $\mathrm{T}$ cells can then migrate to the tumor tissues and recognize tumor antigens presented in complex with HLA class I at the surface of tumor cells, followed by elimination of the latter. ${ }^{22}$

\section{Anti-PD-1 and Anti-PD-L1 Antibodies}

While the anti-CTLA-4 blockade strategy leads to a general enhancement of T-cell priming, antibodies directed against PD-1 and PD-L1 act on T cells that have already been activated, but circulate in the lymph nodes or reside in the tumor microenvironment itself. PD-1 is a surface protein expressed on activated T and B cells while PD-L1 is mostly expressed on APCs, such as macrophages and DCs, and tumor-infiltrating lymphocytes (TILs), but can also be expressed on tumor cells. ${ }^{23}$ In normal physiology, the PD-1/PD-L1 axis represents an important immune checkpoint to prevent immune-mediated tissue damage (Figure 2), and PD-1 knockout mice have shown increased susceptibility 

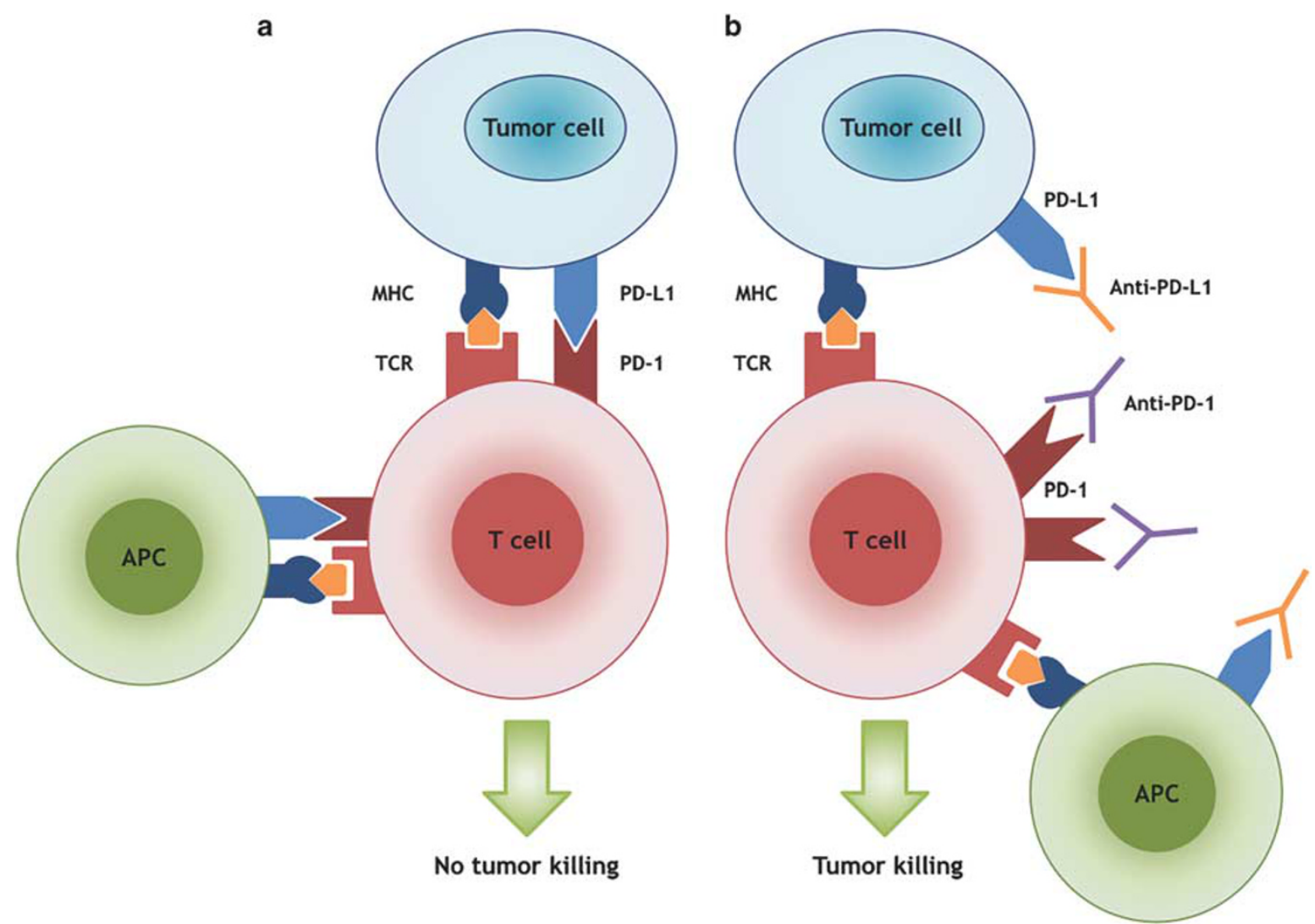

Figure 2 Schematic overview of the mechanism of action of anti-PD-1 and anti-PD-L1 antibodies. Tumor cells can upregulate PD-L1 expression by genetic alterations or chromosomal translocation. INF- $\gamma$ and other cytokines in the tumor microenvironment can also upregulate the expression of PD-L1 on tumor cells and myeloid cells, such as APCs. PD-L1 on these cells binds to PD-1 on active T cells, inhibiting the T-cell receptor-mediated proliferation of the T cells, leading to reduced killing of the tumor cells. CD8 ${ }^{+}$T cells can interact with tumor cells and APCs through MHC class I molecules, whereas $\mathrm{CD}^{+} \mathrm{T}$ cells interact with APCs through MHC class II molecules. (a) Administration of anti-PD-L1 and anti-PD-1 antibodies prevents immune inhibition by the PD-L1 on the tumor cells or myeloid cells and subsequently enhances tumor killing (b).

to the development of autoimmune disorders. ${ }^{24}$ As PD-1 is expressed on exhausted $\mathrm{T}$ cells, anti-PD-1 therapy can reinforce the immune responses of this subset of T cells. ${ }^{25}$ However, PD-1 is also expressed during earlier stages of T-cell activation, where it has an important role in the induction of tolerance against self-antigens and the generation of active cytotoxic T lymphocytes. ${ }^{26}$ Of note, PD-1 knockout mice exhibit autoimmunity with a markedly augmented CD8 proliferation. ${ }^{24}$ Administration of anti-PD-1 or anti-PD-L1 antibodies aims to release this immunological break and enhance the cytotoxic T-cell response, leading to tumor control or elimination.

Various cancer types like non-small-cell lung carcinoma and melanoma exploit this immunosuppressive interaction and show expression of PD-L1. ${ }^{23,27,28}$ For example, the expression of PD-L1 on tumor cells can be the result of genetic alterations. ${ }^{29}$ More often, PD-L1 expression on tumor cells, APCs, and other myeloid cells is induced by IFN- $\gamma$ and other inflammatory cytokines present in the tumor microenvironment. ${ }^{30}$ In addition to the PD-1-mediated immunosuppression, many of the tumor-infiltrating myeloid cells, such as myeloid-derived suppressor cells and tumorassociated macrophages (TAMs), show pro-tumorigenic activity. Some sarcomas secrete factors such as colonystimulating factor-1 (CSF-1) and others to attract and stimulate TAMs, creating an immunosuppressive microenvironment. ${ }^{31,32}$ Recent data suggest that TAMs might facilitate tumor resistance against anti-PD-1 and anti-PD-L1 therapy. ${ }^{33,34}$ To explore the combination of inhibition of TAMs and enhancement of T-cell-mediated immune responses, an ongoing clinical trial is exploring the combined inhibition of the CSF-1 receptor and PD-1 pathway in GIST and other solid tumors (ClinicalTrials.gov Identifier: NCT02452424).

\section{BIOMARKERS FOR IMMUNOTHERAPY IN SARCOMAS}

There is a clear rationale for the use of immune checkpoint inhibitors in sarcomas. Many sarcomas harbor chromosomal translocations resulting in expressed fusion proteins, which can provoke an immunological response. Of note, fusion proteins of synovial sarcoma, clear cell sarcoma, and desmoplasmic small round cell tumor have been 
demonstrated to bind HLA class I molecules. ${ }^{35}$ In one study, an in vitro cytotoxic $\mathrm{T}$-cell response against alveolar rhabdomyosarcoma cells was induced using DCs pulsed with a PAX-FKHR fusion protein breakpoint epitope. ${ }^{36}$ Some sarcomas, such as synovial sarcomas and myxoid liposarcomas, also overexpress cancer/testis antigens like NY-ESO-1, which can also trigger $\mathrm{CD}^{+}{ }^{+}$T-cell-mediated lysis of tumor cells. ${ }^{37,38}$ Treatment with anti-CTLA-4, anti-PD-1, or antiPD-L1 antibodies could enhance $\mathrm{CD}^{+}$T-cell-mediated tumor lysis by skipping the crucial immune checkpoints during T-cell priming, activation, and T-cell-mediated eradication of the tumor. Multiple clinical trials are now investigating the potential of immune checkpoint inhibitors as a treatment for sarcoma patients. The first clinical results from the SARC028 study, where patients with advanced sarcomas were treated with the anti-PD-1 antibody pembrolizumab, showed response in patients with undifferentiated pleomorphic sarcoma, dedifferentiated liposarcoma, synovial sarcoma, osteosarcoma, and dedifferentiated chondrosarcoma. ${ }^{39}$ In a cohort of patients with metastatic sarcoma, Paoluzzi et al ${ }^{40}$ observed partial responses in a dedifferentiated chondrosarcoma, epithelioid sarcoma, and osteosarcoma after treatment with nivolumab. However, in one pilot study including six synovial sarcoma patients, treatment with anti-CTLA-4 antibody did not result in an immunological antitumor response and the disease progressed rapidly in all patients. ${ }^{41}$ Therefore, biomarker identification will be fundamental to improve the selection of sarcomas that will respond to therapy with immune checkpoint inhibitors.

\section{Tumor-Infiltrating Lymphocytes}

The number and type of TILs could serve as a predictive biomarker for treatment with immune checkpoint inhibitors. In a cohort of patients with metastatic melanomas, patients responding to anti-PD-1 treatment were shown to have a higher number of $\mathrm{CD} 8{ }^{+}$TILs before treatment. ${ }^{42}$ This finding clearly indicates that an ongoing antitumor immune response present before treatment is important for the clinical outcome of treatment with anti-PD-1 therapy. Furthermore, in many cancers the presence of TILs often serves as a prognostic factor for patient survival. In patients with colon cancer, higher T-cell infiltration in the tumor was shown to be a predictive biomarker for disease-free survival. ${ }^{43} \mathrm{~A}$ high count of TILs also associated with better overall survival in melanoma patients. ${ }^{44}$ Furthermore, presence of tumorinfiltrating $\mathrm{T}_{\text {reg }}$ cells correlated with a worse prognosis in patients with ovarian cancer and renal cell carcinoma. ${ }^{45,46}$ These findings confirm the active role for infiltrating immune cells in controlling cancer progression. Moreover, depletion of T cells in an osteosarcoma mouse model and in the Swarm rat chondrosarcoma model resulted in a markedly reduced survival rate and an accelerated growth rate, respectively, revealing a function for $\mathrm{CD}^{+} \mathrm{T}$ cells in this experimental setting in slowing sarcoma progression. ${ }^{47,48}$ Although a high number of infiltrating $\mathrm{CD}^{+} \mathrm{T}$ cells does not seem to be a clear prognostic marker for survival in patients with softtissue sarcoma, high expression of PD-1 on TILs and expression of $\mathrm{PD}-\mathrm{L} 1$ correlate with worse survival rates in these patients, suggesting that expression of PD-1 and PD-L1 in soft-tissue sarcomas could inhibit T-cell-mediated control of cancer progression. ${ }^{49,50}$

Mapping of the T-cell infiltrate in the tumor microenvironment and its association with patient survival has been investigated in some specific sarcoma subtypes (Table 1). A high number of tumor-infiltrating $\mathrm{CD}^{+} \mathrm{T}$ cells correlated with improved overall survival in Ewing sarcoma. ${ }^{51}$ In osteosarcoma, PD-L1 expression on tumor cells associated with higher numbers of TILs and poorer survival rate, indicating that the PD-1/PD-L1 axis is an important immune evasion strategy of sarcomas. ${ }^{52}$ PD-L1 expression has also been observed in almost $50 \%$ of dedifferentiated chondrosarcomas and correlated with a higher number of TILs and positive HLA class I expression in tumor cells, providing a rationale for using anti-PD-1 and anti-PD-L1 treatment in this sarcoma subtype. ${ }^{53} \mathrm{~A}$ high number of intratumoral lymphocytes was also observed in EBV-associated leiomyosarcomas and inflammatory myofibroblastic tumors. ${ }^{54,55}$

Table 1 Examples of association between TILs and survival in some sarcoma types

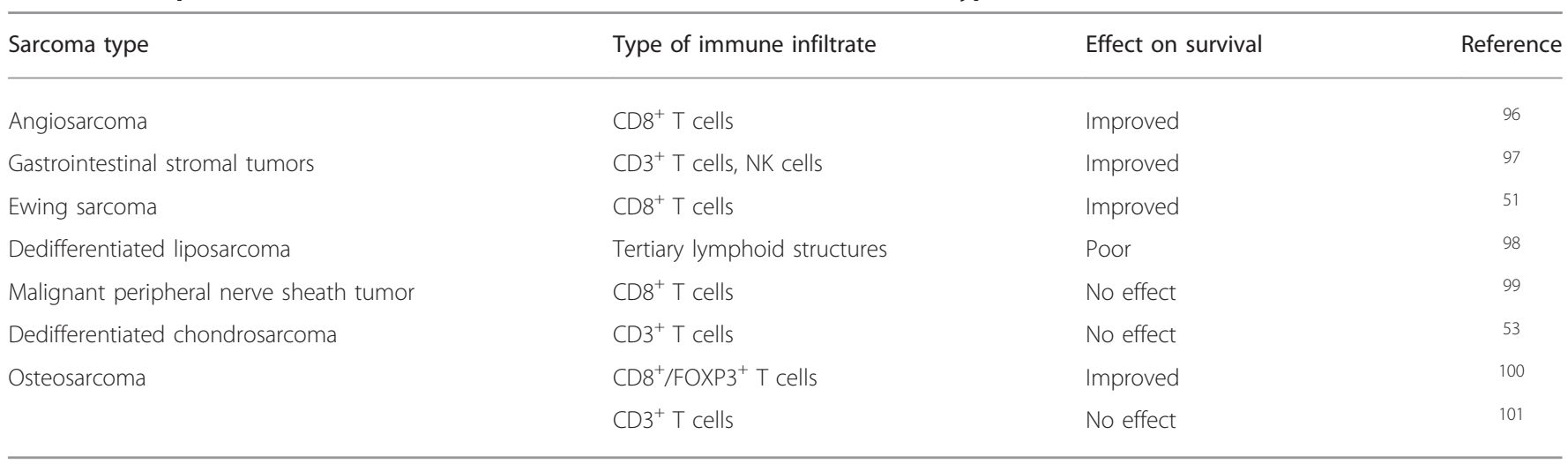


Table 2 Reported PD-L1 expression in some sarcoma subtypes

\begin{tabular}{lcc}
\hline Sarcoma subtype & Positive cases (\%) & Reference \\
\hline Angiosarcoma & $50-80$ & 50,65 \\
Chondrosarcoma & $41-75$ & 53,65 \\
Ewing sarcoma & $29-67$ & $50,65,69$ \\
Leiomyosarcoma & $32-70$ & 50,65 \\
Malignant peripheral nerve sheath tumor & $17-67$ & $50,65,99$ \\
Osteosarcoma & $28-57$ & 65,101 \\
Rhabdomyosarcoma & $38-63$ & 65,69 \\
Synovial sarcoma & $25-75$ & $50,65,69$ \\
Dedifferentiated liposarcoma & $67-82$ & 50,65 \\
Gastrointestinal stromal tumor & 29 & 102 \\
\hline
\end{tabular}

Pollack et $a l^{56}$ used gene expression profiling to measure the amount of T-cell infiltration and found a higher degree of $\mathrm{T}$-cell infiltration in undifferentiated pleomorphic sarcomas and leiomyosarcomas, and a low degree in synovial sarcomas. Interestingly, they also showed that the degree of $\mathrm{T}$-cell infiltration and clonality significantly correlated with PD-1 and PD-L1 expression in all investigated sarcoma subtypes, suggesting that sarcomas with a high number of TILs might be more suitable for anti-PD-1 and anti-PD-L1 therapy than sarcomas with a low degree of T-cell infiltration.

\section{Expression of PD-1 and PD-L1}

Expression of PD-1 and PD-L1 in the tumor microenvironment might be an important predictive biomarker for antiPD-1 therapies. In one of the first clinical trials with antiPD-1 antibodies, tumor samples were immunohistochemically stained for PD-L1 before treatment. Thirty-six percent of the patients with PD-L1-positive tumors had an objective response while none of the patients with PD-L1-negative tumors responded to therapy. ${ }^{6}$ However, in a large cohort of patients with advanced melanoma, patients with a PD-L1positive tumor showed an objective response rate of $57.5 \%$ to treatment with an anti-PD-1 antibody, whereas patients with a PD-L1-negative tumor showed an objective response rate of $41.3 \% .{ }^{57}$ These findings suggest that PD-L1 expression might not always be a clear-cut biomarker for the response to antiPD-1 therapy.

PD-L1 can be overexpressed in cancer cells as result of genetic alterations. In diffuse large B-cell lymphomas, the $P D-L 1$ gene was shown to be translocated and placed under the regulation of a different promotor, notably the immunoglobulin heavy-chain locus, leading to upregulation of PD-L1 expression..$^{29}$ Loss of PTEN in colorectal cancer was also shown to result in increased expression of PD-L1. ${ }^{58}$ Interestingly, some soft-tissue sarcomas, such as liposarcomas and leiomyosarcomas, harbor genetic mutations in PTEN and loss of PTEN has recently be shown to associate with resistance to anti-PD-1 therapy in metastatic uterine leiomyosarcoma. ${ }^{59-64}$

As already discussed above, the expression of PD-L1 is a frequently observed immune evasion strategy of certain sarcomas. So far, several studies have assessed PD-L1 expression in different sarcoma subtypes using immunohistochemistry (Table 2). In a large analysis of over 2000 sarcomas, $\sim 50 \%$ of all sarcomas displayed expression of PDL1 with immunohistochemistry. Notably, PD-L1 expression was observed in leiomyosarcomas, chondrosarcomas, liposarcomas, and undifferentiated pleomorphic sarcoma using immunohistochemistry with an anti-PD-L1 antibody. ${ }^{65}$ Frequent expression of PD-1 and PD-L1 was also observed in synovial sarcoma and angiosarcoma. ${ }^{50}$ In a cohort of 38 osteosarcoma tumor specimens PD-L1 expression was detected with RT-PCR in 32 cases of which 9 had a relative high PD-L1 mRNA expression level. ${ }^{66}$ In HHV8-associated Kaposi sarcoma, PD-L1 expression has also been demonstrated in a large subset of cases. ${ }^{67,68}$ Another study could detect PD-L1 expression in the dedifferentiated chondrosarcoma subtype, but not in the conventional, clear cell or mesenchymal chondrosarcoma subtype. ${ }^{53}$

Although these studies provide a rough estimate of the percentage of PD-L1-expressing sarcomas, they also highlight some discrepancies. ${ }^{50,65,69}$ This lack of reproducibility can be partially explained by the small number of cases and variable cutoff points for PD-L1-positivity. Moreover, the difference could also be explained by different types of antibodies used for the immunohistochemistry of PD-L1. Therefore, assessment of PD-L1 expression in sarcomas with immunohistochemistry is not, so far, a reliable predictive biomarker to preselect patients for treatment with immune checkpoint inhibitors and efforts should be made to standardize this procedure.

\section{Mutational Load}

Next-generation sequencing technologies have made it possible to comprehensively detect somatic mutations in individual tumors and to reveal mutational signature profiles. These techniques therefore provide a novel powerful tool to unravel the underlying genetic pathogenesis of sarcomas. The mutational burden of a tumor can be predictive for the outcome of treatment with immune checkpoint inhibitors. Snyder et al. ${ }^{70}$ showed that treatment with ipilimumab, an anti-CTLA-4 antibody, was significantly more effective in patients with melanomas carrying more than 100 mutations per coding genome when compared to patients with $<100$ mutations in their tumors. For non-small-cell lung cancer, patients with a high nonsynonymous mutation burden were also more likely to show improved clinical benefit when treated with an anti-PD-1 antibody than patients with a low nonsynonymous mutation burden. ${ }^{71}$ These findings support that a higher mutational load probably translates into an increased probability that neoantigens are recognized by the immune system. In line with these findings, the association 
Table 3 Examples of mutational load in different sarcoma types reported by sequencing studies

\begin{tabular}{lcclc}
\hline Sarcoma type & Reported mutations & Mutations/Mbp & Determined mutation rate \\
\hline Ewing sarcoma & 6 & 0.15 & Average in protein-coding sequences \\
Osteosarcoma & $5-103$ & 1.15 & Mean mutation rate, whole genome \\
Solitary fibrous tumors & $12-41$ & 0.66 & Median mutation rate, whole exome \\
Angiosarcoma & - & $0.7-2.2$ & Whole genome \\
Uterine leiomyosarcoma & $240-779$ & - & Exome \\
Malignant peripheral nerve sheath tumor & $14-208$ & - & Whole genome \\
Chondrosarcoma & $1-115$ & - & Exome \\
Rhabdomyosarcoma & 24 & - & Whole exome \\
Well-differentiated liposarcoma & $16-71$ & - & Whole exome \\
Dedifferentiated liposarcoma & $24-56$ & - & Whole exome \\
Myxoid liposarcoma & $15-33$ & & Whole exome \\
\hline
\end{tabular}

between response to ipilimumab treatment, overall mutational, and neoantigen load has been shown in melanoma patients. ${ }^{72,73}$

Although the application of next-generation sequencing in sarcomas is a developing research field, recent studies suggest that some sarcomas are driven by an intermediate mutational load (Table 3). For example, a high median frequency of somatic mutations of $\sim 14$ mutations per megabase pair (Mbp) has been reported for melanoma and a low median frequency of 0.37 mutations per Mbp as reported for acute myeloid leukemia. Compared with these reported mutation rates, the mean mutation rate of 1.15 mutations per Mbp found in a cohort of 20 high-grade intramedullary osteosarcomas is an intermediate rate, which is-although difficult to compare, as often different pipelines for analysis have been utilized-roughly similar to the median mutational rate of breast cancer shown by Alexandrov and colleagues. ${ }^{74-77}$ Furthermore, this cohort of osteosarcomas showed a range in the mutational rate between 0.49 and 3.99 mutations per $\mathrm{Mbp}$, suggesting that some individual cases could benefit more from treatment with immune checkpoint inhibitors due to their higher-than-average mutational rate. ${ }^{77}$ Ewing sarcoma has a reported mutation rate of 0.15 mutations per Mbp of coding sequence in one study and a mutation rate of 0.62 per Mbp in another study, both indicating that the mutational rate of this translocation-driven tumor can be categorized with other cancer types with a low mutational rate..$^{75,78,79}$ Exome sequencing of uterine leiomyosarcomas revealed a mean of 373 somatic mutations per sample, whereas the mutation burden in chondrosarcoma has been shown to range from 1 to 115 somatic mutations. ${ }^{80,81}$ In another study, exome sequencing of rhabdomyosarcoma cases revealed a mean number of mutations of 24.0 mutations per sample in a primary tumor, while metastatic tumors showed 43.3 mutations per sample and relapsed tumors 42.0 mutations

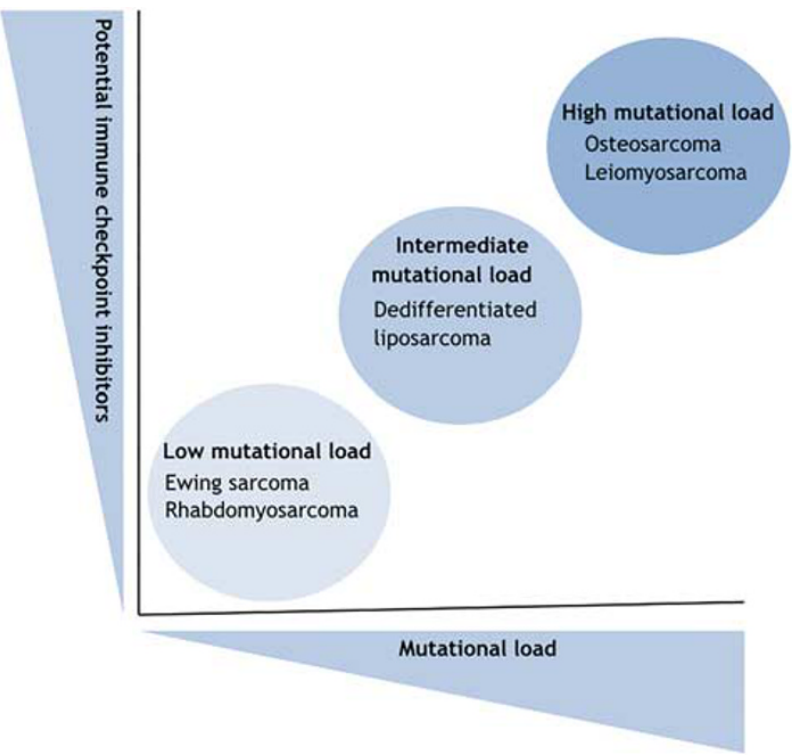

Figure 3 Mutational load and potential for immune checkpoint inhibitors in sarcomas. Some sarcomas such as Ewing sarcoma are mainly driven by specific translocations and do not display a high mutational profile, while other sarcomas such as osteosarcoma show a higher mutation burden and a more pleomorphic histology. A higher mutational load increases the change of neoantigen formation and enhances the immunogenicity of the tumor.

per sample, although these differences were not statistically significant. ${ }^{82}$ Epithelioid sarcomas have a coding somatic point mutation rate similar to that of ovarian carcinoma, indicating a relevant mutation rate in this sarcoma type, which could be beneficial for treatment with immune checkpoint inhibitors. ${ }^{83,84}$ In 12 liposarcoma specimens of different subtypes, a total of 377 potential somatic mutations were detected of which 91\% was validated with Sanger sequencing. ${ }^{85}$ Although some next-generation sequencing 
studies have reported the mutation burden of certain sarcomas, it is still very difficult to directly compare the mutational load of different sarcomas based on these studies. However, as the field of next-generation sequencing is rapidly developing, the mutational load of more sarcomas can soon be unraveled and compared with other cancers, which will be a crucial step in further exploring the potential of treatment with immune checkpoint inhibitors in sarcomas (Figure 3).

\section{DNA Mismatch Repair System}

The proteins of the DNA mismatch repair (MMR) system are crucial in restoring incorporated mismatched bases during replication. As such, MMR-deficient tumors have been shown to be genetically instable tumors with a relative higher mutational load. For example, MMR-deficient colorectal cancers often show a high mutational rate, microsatellite instability, and a higher degree of TILs, suggesting an ongoing immune response against the tumor. ${ }^{86,87}$ The higher degree of tumor infiltration by lymphocytes and the higher mutational load suggest that MMR-deficient tumors could be more suitable for the treatment with immune checkpoint inhibitors than MMR-proficient tumors. In a phase 2 clinical trial published by Le et al, ${ }^{8} 41$ patients with metastatic carcinoma were treated with an anti-PD-1 antibody, and in line with their hypothesis, they found a significantly increased progression-free survival rate and overall survival in patients with MMR-deficient colorectal cancer compared to patients with MMR-proficient colorectal cancer.

The outcome of this clinical trial provides clear evidence for the use of immune checkpoint inhibitors in MMRdeficient cancers. Although evidence is often conflicting, in some cases, certain sarcomas can display defects in the MMR system. ${ }^{88}$ Twenty-one percent of the cases in a cohort of uterine carcinosarcomas were shown to have a defective MMR system based on a microsatellite instability phenotype and a small percentage of leiomyosarcomas are also deficient in MMR. ${ }^{89,90}$ Ongoing research could shed more light on MMR deficiency in sarcomas, as its role still remains to be elucidated.

\section{Biomarkers for Hyperprogression}

Very recently, the phenomenon of hyperprogression was described in cohorts of patients that were treated with antiPD-1/PD-L1 and anti-CTLA-4 antibodies. ${ }^{91,92}$ In some of these patients, treatment with these immune checkpoint inhibitors accelerated the growth of their tumors. This hyperprogression was shown to correlate with amplification of the MDM2 and MDM4 genes and mutations in the EGFR gene. ${ }^{92}$ In a large survey for MDM2 amplification in a variety of tumor types, MDM2 amplification was found to be most prevalent in sarcomas. ${ }^{93}$ Amplification of the 12q13-15 region, including the $M D M 2$ gene is the hallmark of welldifferentiated and dedifferentiated liposarcoma, as well as parosteal osteosarcoma and is used as diagnostic marker. ${ }^{94}$ In addition, other sarcomas also display MDM2 amplification in a low percentage of the tumors, including conventional osteosarcomas and malignant peripheral nerve sheath tumors. ${ }^{95}$ These studies suggest that for these sarcoma subtypes treatment with immune checkpoint inhibitors must be carefully considered.

\section{CONCLUSIONS}

Immune checkpoint inhibitors have accelerated the immunotherapy revolution in oncology. As metastatic sarcomas have limited options for treatment, these therapeutics could be an interesting novel treatment option. While some first promising results in sarcoma are now being published, several clinical trials are still ongoing. A selection of sarcomas that are most suitable for treatment with immune checkpoint inhibitors can be guided by recently proposed biomarkers in other cancers. As a correlation between TILs and PD-L1 has been found in certain sarcomas, such as osteosarcoma and dedifferentiated chondrosarcoma, PD-L1 expression in these sarcomas can be considered an immunosuppressive tool to prevent TILs from eliminating tumor cells. This provides a strong rationale for therapy with anti-PD-1 or anti-PD-L1 antibodies in sarcomas with a high degree of TILs or PD-L1 expression. Furthermore, some sarcomas, such as osteosarcoma and epithelioid sarcomas, show an intermediate mutation burden when compared with other cancer types. In addition, although some sarcomas display a higher mutational load than others, individual cases with a relatively higher mutational load can often be identified in a cohort of patients. As a high mutation burden has been associated with a higher neoantigen load and a better survival rate after administration of immune checkpoint inhibitors, patients with a hypermutated sarcoma could benefit from treatment with immune checkpoint inhibitors. Larger exome-wide and genome-wide sequencing studies could provide novel insights in the mutational landscape of sarcomas and can help guiding the selection of sarcoma types for treatment with immune checkpoint inhibitors.

\section{DISCLOSURE/CONFLICT OF INTEREST}

The authors declare no conflict of interest.

1. Burningham $Z$, Hashibe $M$, Spector $L$, et al. The epidemiology of sarcoma. Clin Sarcoma Res 2012;2:14.

2. Italiano A, Mathoulin-Pelissier S, Cesne AL, et al. Trends in survival for patients with metastatic soft-tissue sarcoma. Cancer 2011;117: 1049-1054.

3. Hodi FS, O'Day SJ, McDermott DF, et al. Improved survival with ipilimumab in patients with metastatic melanoma. $\mathrm{N}$ Engl J Med 2010;363:711-723.

4. Maio M, Grob JJ, Aamdal S, et al. Five-year survival rates for treatment-naive patients with advanced melanoma who received ipilimumab plus dacarbazine in a phase III trial. J Clin Oncol 2015;33: 1191-1196.

5. Ansell SM, Lesokhin AM, Borrello I, et al. PD-1 blockade with nivolumab in relapsed or refractory Hodgkin's lymphoma. N Engl J Med 2015;372:311-319.

6. Topalian SL, Hodi FS, Brahmer JR, et al. Safety, activity, and immune correlates of anti-PD-1 antibody in cancer. N Engl J Med 2012;366: 2443-2454. 
7. Hamid O, Robert C, Daud A, et al. Safety and tumor responses with lambrolizumab (anti-PD-1) in melanoma. N Engl J Med 2013;369: 134-144.

8. Le DT, Uram JN, Wang $\mathrm{H}$, et al. PD-1 blockade in tumors with mismatch-repair deficiency. N Engl J Med 2015;372:2509-2520.

9. Brahmer JR, Tykodi SS, Chow LQ, et al. Safety and activity of anti-PDL1 antibody in patients with advanced cancer. N Engl J Med 2012;366: 2455-2465.

10. Powles T, Eder JP, Fine GD, et al. MPDL3280A (anti-PD-L1) treatment leads to clinical activity in metastatic bladder cancer. Nature 2014;515:558-562

11. Philips GK, Atkins M. Therapeutic uses of anti-PD-1 and anti-PD-L1 antibodies. Int Immunol 2015;27:39-46.

12. Lim J, Poulin NM, Nielsen TO. New strategies in sarcoma: linking genomic and immunotherapy approaches to molecular subtype. Clin Cancer Res 2015;21:4753-4759.

13. Topalian SL, Taube JM, Anders RA, et al. Mechanism-driven biomarkers to guide immune checkpoint blockade in cancer therapy. Nat Rev Cancer 2016;16:275-287.

14. Chen PL, Roh W, Reuben A, et al. Analysis of immune signatures in longitudinal tumor samples yields insight into biomarkers of response and mechanisms of resistance to immune checkpoint blockade. Cancer Discov 2016;6:827-837.

15. Burnet M. Cancer; a biological approach. I. The processes of control. Br Med J 1957;1:779-786.

16. Preynat-Seauve $\mathrm{O}$, Schuler $\mathrm{P}$, Contassot $\mathrm{E}$, et al. Tumor-infiltrating dendritic cells are potent antigen-presenting cells able to activate T cells and mediate tumor rejection. J Immunol 2006;176:61-67.

17. Santana MA, Esquivel-Guadarrama F. Cell biology of T cell activation and differentiation. Int Rev Cytol 2006;250:217-274.

18. Judge TA, Tang A, Turka LA. Immunosuppression through blockade of CD28:B7-mediated costimulatory signals. Immunol Res 1996;15: 38-49.

19. Wing $K$, Onishi $Y$, Prieto-Martin $P$, et al. CTLA-4 control over Foxp3+ regulatory $T$ cell function. Science 2008;322:271-275.

20. Tivol EA, Borriello F, Schweitzer AN, et al. Loss of CTLA-4 leads to massive lymphoproliferation and fatal multiorgan tissue destruction, revealing a critical negative regulatory role of CTLA-4. Immunity 1995;3:541-547.

21. Waterhouse P, Penninger JM, Timms E, et al. Lymphoproliferative disorders with early lethality in mice deficient in Ctla-4. Science 1995;270:985-988.

22. Weber JS, Hamid O, Chasalow SD, et al. Ipilimumab increases activated $\mathrm{T}$ cells and enhances humoral immunity in patients with advanced melanoma. J Immunother 2012;35:89-97.

23. Chen Z, Mei J, Liu L, et al. PD-L1 expression is associated with advanced non-small cell lung cancer. Oncol Lett 2016;12:921-927.

24. Nishimura $H$, Nose $M$, Hiai $H$, et al. Development of lupus-like autoimmune diseases by disruption of the PD-1 gene encoding an ITIM motif-carrying immunoreceptor. Immunity 1999;11:141-151.

25. Yi JS, Cox MA, Zajac AJ. T-cell exhaustion: characteristics, causes and conversion. Immunology 2010;129:474-481.

26. Goldberg MV, Maris $\mathrm{CH}$, Hipkiss EL, et al. Role of PD-1 and its ligand, B7-H1, in early fate decisions of CD8 T cells. Blood 2007;110: 186-192.

27. Scheel $A H$, Ansen S, Schultheis AM, et al. PD-L1 expression in nonsmall cell lung cancer: Correlations with genetic alterations. Oncoimmunology 2016;5:e1131379.

28. Massi D, Brusa D, Merelli B, et al. The status of PD-L1 and tumorinfiltrating immune cells predict resistance and poor prognosis in BRAFi-treated melanoma patients harboring mutant BRAFV600. Ann Oncol 2015;26:1980-1987.

29. Georgiou K, Chen L, Berglund M, et al. Genetic basis of PD-L1 overexpression in diffuse large B-cell lymphomas. Blood 2016;127: 3026-3034.

30. Santarpia M, Karachaliou N. Tumor immune microenvironment characterization and response to anti-PD-1 therapy. Cancer Biol Med 2015;12:74-78.

31. Ganjoo KN, Witten D, Patel M, et al. The prognostic value of tumorassociated macrophages in leiomyosarcoma: a single institution study. Am J Clin Oncol 2011;34:82-86.

32. West RB, Rubin BP, Miller MA, et al. A landscape effect in tenosynovial giant-cell tumor from activation of CSF1 expression by a translocation in a minority of tumor cells. Proc Natl Acad Sci USA 2006;103: 690-695.

33. Arlauckas SP, Garris CS, Kohler $\mathrm{RH}$, et al. In vivo imaging reveals a tumor-associated macrophage-mediated resistance pathway in antiPD-1 therapy. Sci Transl Med 2017;9:389.

34. Toulmonde M, Penel N, Adam J, et al. Use of PD-1 targeting, macrophage infiltration, and IDO pathway activation in sarcomas: a phase 2 clinical trial. JAMA Oncol 2017.

35. Worley BS, van den Broeke LT, Goletz TJ, et al. Antigenicity of fusion proteins from sarcoma-associated chromosomal translocations. Cancer Res 2001;61:6868-6875.

36. van den Broeke LT, Pendleton CD, Mackall C, et al. Identification and epitope enhancement of a PAX-FKHR fusion protein breakpoint epitope in alveolar rhabdomyosarcoma cells created by a tumorigenic chromosomal translocation inducing CTL capable of lysing human tumors. Cancer Res 2006;66:1818-1823.

37. Ayyoub M, Taub RN, Keohan ML, et al. The frequent expression of cancer/testis antigens provides opportunities for immunotherapeutic targeting of sarcoma. Cancer Immun 2004;4:7.

38. Endo $M$, de Graaff MA, Ingram DR, et al. NY-ESO-1 (CTAG1B) expression in mesenchymal tumors. Mod Pathol 2015;28: 587-595.

39. Burgess MA, Bolejack V, Tine BAV, et al. Multicenter phase II study of pembrolizumab (P) in advanced soft tissue (STS) and bone sarcomas (BS): final results of SARC028 and biomarker analyses. J Clin Oncol 2017;35(15_suppl):11008-11008.

40. Paoluzzi L, Cacavio A, Ghesani M, et al. Response to anti-PD1 therapy with nivolumab in metastatic sarcomas. Clin Sarcoma Res 2016;6:24.

41. Maki RG, Jungbluth AA, Gnjatic $S$, et al. A pilot study of anti-CTLA4 antibody ipilimumab in patients with synovial sarcoma. Sarcoma 2013;2013:168145.

42. Tumeh PC, Harview CL, Yearley JH, et al. PD-1 blockade induces responses by inhibiting adaptive immune resistance. Nature 2014;515:568-571.

43. Galon J, Costes A, Sanchez-Cabo F, et al. Type, density, and location of immune cells within human colorectal tumors predict clinical outcome. Science 2006;313:1960-1964.

44. Clemente CG, Mihm Jr. MC, Bufalino R, et al. Prognostic value of tumor infiltrating lymphocytes in the vertical growth phase of primary cutaneous melanoma. Cancer 1996;77:1303-1310.

45. Wolf $\mathrm{D}$, Wolf $\mathrm{AM}$, Rumpold $\mathrm{H}$, et al. The expression of the regulatory $\mathrm{T}$ cell-specific forkhead box transcription factor FoxP3 is associated with poor prognosis in ovarian cancer. Clin Cancer Res 2005;11: 8326-8331.

46. Siddiqui SA, Frigola X, Bonne-Annee S, et al. Tumor-infiltrating Foxp3CD4+CD25+ T cells predict poor survival in renal cell carcinoma. Clin Cancer Res 2007;13:2075-2081.

47. Lussier DM, O'Neill L, Nieves LM, et al. Enhanced T-cell immunity to osteosarcoma through antibody blockade of PD-1/PD-L1 interactions. J Immunother 2015;38:96-106.

48. Simard FA, Richert I, Vandermoeten A, et al. Description of the immune microenvironment of chondrosarcoma and contribution to progression. Oncoimmunology 2017;6:e1265716.

49. Sorbye SW, Kilvaer TK, Valkov A, et al. Prognostic impact of peritumoral lymphocyte infiltration in soft tissue sarcomas. BMC Clin Pathol 2012;12:5.

50. Kim JR, Moon YJ, Kwon KS, et al. Tumor infiltrating PD1-positive lymphocytes and the expression of PD-L1 predict poor prognosis of soft tissue sarcomas. PLoS ONE 2013;8:e82870.

51. Berghuis D, Santos SJ, Baelde HJ, et al. Pro-inflammatory chemokinechemokine receptor interactions within the Ewing sarcoma microenvironment determine CD8(+) T-lymphocyte infiltration and affect tumour progression. J Pathol 2011;223:347-357.

52. Koirala P, Roth ME, Gill J, et al. Immune infiltration and PD-L1 expression in the tumor microenvironment are prognostic in osteosarcoma. Sci Rep 2016;6:30093.

53. Kostine M, Cleven AH, de Miranda NF, et al. Analysis of PD-L1, T-cell infiltrate and HLA expression in chondrosarcoma indicates potential for response to immunotherapy specifically in the dedifferentiated subtype. Mod Pathol 2016;29:1028-1037.

54. Deyrup AT, Lee VK, Hill CE, et al. Epstein-Barr virus-associated smooth muscle tumors are distinctive mesenchymal tumors 
reflecting multiple infection events: a clinicopathologic and molecular analysis of 29 tumors from 19 patients. Am J Surg Pathol 2006:30:75-82.

55. Behranwala KA, Straker $P$, Wan $A$, et al. Inflammatory myofibroblastic tumour of the gallbladder. World J Surg Oncol 2005;3:24.

56. Pollack $\mathrm{SM}, \mathrm{He} \mathrm{Q}$, Yearley $\mathrm{JH}$, et al. T-cell infiltration and clonality correlate with programmed cell death protein 1 and programmed death-ligand 1 expression in patients with soft tissue sarcomas. Cancer 2017;123:3291-3304.

57. Larkin J, Chiarion-Sileni V, Gonzalez R, et al. Combined nivolumab and ipilimumab or monotherapy in untreated melanoma. N Engl J Med 2015;373:23-34.

58. Song $M$, Chen $D$, Lu B, et al. PTEN loss increases PD-L1 protein expression and affects the correlation between PD-L1 expression and clinical parameters in colorectal cancer. PLoS ONE 2013;8: e65821.

59. Kawaguchi K, Oda Y, Saito T, et al. Genetic and epigenetic alterations of the PTEN gene in soft tissue sarcomas. Hum Pathol 2005;36: 357-363.

60. Saito T, Oda Y, Kawaguchi K, et al. PTEN/MMAC1 gene mutation is a rare event in soft tissue sarcomas without specific balanced translocations. Int J Cancer 2003;104:175-178.

61. Yin L, Liu CX, Nong WX, et al. Mutational analysis of p53 and PTEN in soft tissue sarcoma. Mol Med Rep 2012;5:457-461.

62. Puzio-Kuter AM, Laddha SV, Castillo-Martin M, et al. Involvement of tumor suppressors PTEN and $\mathrm{p} 53$ in the formation of multiple subtypes of liposarcoma. Cell Death Differ 2015;22: 1785-1791.

63. Grossmann AH, Layfield LJ, Randall RL. Classification, molecular characterization, and the significance of pten alteration in leiomyosarcoma. Sarcoma 2012;2012:380896.

64. George S, Miao D, Demetri GD, et al. Loss of PTEN is associated with resistance to anti-PD-1 checkpoint blockade therapy in metastatic uterine leiomyosarcoma. Immunity 2017;46:197-204.

65. Movva S, Wen W, Chen W, et al. Multi-platform profiling of over 2000 sarcomas: identification of biomarkers and novel therapeutic targets. Oncotarget 2015;6:12234-12247.

66. Shen JK, Cote GM, Choy E, et al. Programmed cell death ligand 1 expression in osteosarcoma. Cancer Immunol Res 2014;2: 690-698.

67. Chen BJ, Chapuy B, Ouyang J, et al. PD-L1 expression is characteristic of a subset of aggressive B-cell lymphomas and virus-associated malignancies. Clin Cancer Res 2013;19:3462-3473.

68. Paydas S, Bagir EK, Deveci MA, et al. Clinical and prognostic significance of PD-1 and PD-L1 expression in sarcomas. Med Oncol 2016;33:93.

69. Kim C, Kim EK, Jung $H$, et al. Prognostic implications of PD-L1 expression in patients with soft tissue sarcoma. BMC Cancer 2016;16: 434.

70. Snyder A, Makarov V, Merghoub T, et al. Genetic basis for clinical response to CTLA-4 blockade in melanoma. N Engl J Med 2014;371: 2189-2199.

71. Rizvi NA, Hellmann MD, Snyder A, et al. Cancer immunology. Mutational landscape determines sensitivity to PD-1 blockade in non-small cell lung cancer. Science 2015;348:124-128.

72. Van Allen EM, Miao D, Schilling B, et al. Genomic correlates of response to CTLA-4 blockade in metastatic melanoma. Science 2015;350:207-211.

73. Schumacher TN, Schreiber RD. Neoantigens in cancer immunotherapy. Science 2015;348:69-74.

74. Alexandrov LB, Nik-Zainal S, Wedge DC, et al. Signatures of mutational processes in human cancer. Nature 2013;500:415-421.

75. Lawrence MS, Stojanov $P$, Polak $P$, et al. Mutational heterogeneity in cancer and the search for new cancer-associated genes. Nature 2013;499:214-218.

76. Davar D, Lin Y, Kirkwood JM. Unfolding the mutational landscape of human melanoma. J Invest Dermatol 2015;135:659-662.

77. Chen X, Bahrami A, Pappo A, et al. Recurrent somatic structural variations contribute to tumorigenesis in pediatric osteosarcoma. Cell Rep 2014;7:104-112.

78. Brohl AS, Solomon DA, Chang W, et al. The genomic landscape of the Ewing Sarcoma family of tumors reveals recurrent STAG2 mutation. PLoS Genet 2014;10:e1004475.
79. Crompton BD, Stewart C, Taylor-Weiner A, et al. The genomic landscape of pediatric Ewing sarcoma. Cancer Discov 2014;4: 1326-1341.

80. Makinen $N$, Aavikko $M$, Heikkinen $T$, et al. Exome sequencing of uterine leiomyosarcomas identifies frequent mutations in TP53, ATRX, and MED12. PLoS Genet 2016;12:e1005850.

81. Tarpey PS, Behjati S, Cooke SL, et al. Frequent mutation of the major cartilage collagen gene COL2A1 in chondrosarcoma. Nat Genet 2013;45:923-926.

82. Seki $M$, Nishimura $R$, Yoshida $K$, et al. Integrated genetic and epigenetic analysis defines novel molecular subgroups in rhabdomyosarcoma. Nat Commun 2015;6:7557.

83. Jamshidi F, Bashashati A, Shumansky K, et al. The genomic landscape of epithelioid sarcoma cell lines and tumours. J Pathol 2016;238: 63-73.

84. Greenman C, Stephens $\mathrm{P}$, Smith R, et al. Patterns of somatic mutation in human cancer genomes. Nature 2007;446:153-158.

85. Kanojia D, Nagata $Y$, Garg $M$, et al. Genomic landscape of liposarcoma. Oncotarget 2015;6:42429-42444.

86. Gologan A, Sepulveda AR. Microsatellite instability and DNA mismatch repair deficiency testing in hereditary and sporadic gastrointestinal cancers. Clin Lab Med 2005;25:179-196.

87. Tougeron D, Fauquembergue $E$, Rouquette $A$, et al. Tumor-infiltrating lymphocytes in colorectal cancers with microsatellite instability are correlated with the number and spectrum of frameshift mutations. Mod Pathol 2009;22:1186-1195.

88. Monument MJ, Lessnick SL, Schiffman JD, et al. Microsatellite instability in sarcoma: fact or fiction? ISRN Oncol 2012;2012: 473146.

89. Taylor NP, Zighelboim I, Huettner PC, et al. DNA mismatch repair and TP53 defects are early events in uterine carcinosarcoma tumorigenesis. Mod Pathol 2006;19:1333-1338.

90. Hoang LN, Ali RH, Lau S, et al. Immunohistochemical survey of mismatch repair protein expression in uterine sarcomas and carcinosarcomas. Int J Gynecol Pathol 2014;33:483-491.

91. Champiat $S$, Dercle L, Ammari $S$, et al. Hyperprogressive disease is a new pattern of progression in cancer patients treated by anti-PD-1/ PD-L1. Clin Cancer Res 2017;23:1920-1928.

92. Kato S, Goodman AM, Walavalkar V, et al. Hyper-progressors after immunotherapy: analysis of genomic alterations associated with accelerated growth rate. Clin Cancer Res 2017;23:4242-4250.

93. Momand J, Jung D, Wilczynski S, et al. The MDM2 gene amplification database. Nucleic Acids Res 1998:26:3453-3459.

94. Aleixo PB, Hartmann AA, Menezes IC, et al. Can MDM2 and CDK4 make the diagnosis of well differentiated/dedifferentiated liposarcoma? An immunohistochemical study on 129 soft tissue tumours. J Clin Pathol 2009;62:1127-1135.

95. Nakayama T, Toguchida J, Wadayama B, et al. MDM2 gene amplification in bone and soft-tissue tumors: association with tumor progression in differentiated adipose-tissue tumors. Int J Cancer 1995;64:342-346.

96. Fujii $\mathrm{H}$, Arakawa $\mathrm{A}$, Utsumi $\mathrm{D}$, et al. CD8(+) tumor-infiltrating lymphocytes at primary sites as a possible prognostic factor of cutaneous angiosarcoma. Int J Cancer 2014;134:2393-2402.

97. Rusakiewicz $S$, Semeraro $M$, Sarabi $M$, et al. Immune infiltrates are prognostic factors in localized gastrointestinal stromal tumors. Cancer Res 2013;73:3499-3510.

98. Tseng WW, Malu $S$, Zhang $M$, et al. Analysis of the intratumoral adaptive immune response in well differentiated and dedifferentiated retroperitoneal liposarcoma. Sarcoma 2015;2015: 547460.

99. Shurell E, Singh AS, Crompton JG, et al. Characterizing the immune microenvironment of malignant peripheral nerve sheath tumor by PD-L1 expression and presence of CD8+ tumor infiltrating lymphocytes. Oncotarget 2016;7:64300-64308.

100. Fritzsching B, Fellenberg J, Moskovszky L, et al. CD8+/FOXP3+-ratio in osteosarcoma microenvironment separates survivors from nonsurvivors: a multicenter validated retrospective study. Oncoimmunology 2015;4:e990800.

101. Sundara YT, Kostine M, Cleven AH, et al. Increased PD-L1 and T-cell infiltration in the presence of HLA class I expression in metastatic high-grade osteosarcoma: a rationale for T-cell-based immunotherapy. Cancer Immunol Immunother 2017;66:119-128. 
102. D'Angelo SP, Shoushtari AN, Agaram NP, et al. Prevalence of tumorinfiltrating lymphocytes and PD-L1 expression in the soft tissue sarcoma microenvironment. Hum Pathol 2015;46:357-365.

103. Chmielecki J, Crago AM, Rosenberg M, et al. Whole-exome sequencing identifies a recurrent NAB2-STAT6 fusion in solitary fibrous tumors. Nat Genet 2013;45:131-132.
104. Behjati S, Tarpey PS, Sheldon $\mathrm{H}$, et al. Recurrent PTPRB and PLCG1 mutations in angiosarcoma. Nat Genet 2014;46: 376-379.

105. Zhang M, Wang $Y$, Jones S, et al. Somatic mutations of SUZ12 in malignant peripheral nerve sheath tumors. Nat Genet 2014;46: 1170-1172. 